ARTICLE

\title{
Structure of the $\mathrm{G}$ protein chaperone and guanine nucleotide exchange factor Ric-8A bound to Goi1
}

Levi J. McClelland ${ }^{1,11}$, Kaiming Zhang (1) 2,11, Tung-Chung Mou1,3,11, Jake Johnston ${ }^{1,3}$, Cindee Yates-Hansen', Shanshan Li (10) 2, Celestine J. Thomas 1,10, Tzanko I. Doukov (10) 4, Sarah Triest ${ }^{5,6}$, Alexandre Wohlkonig,6, Gregory G. Tall ${ }^{7}$, Jan Steyaert (iD ${ }^{5,6}$, Wah Chiu ${ }^{2,8 凶}$ \& Stephen R. Sprang (1) 1,3,9凶

Ric-8A is a cytosolic Guanine Nucleotide exchange Factor (GEF) that activates heterotrimeric $G$ protein alpha subunits $(G \alpha)$ and serves as an essential $G \alpha$ chaperone. Mechanisms by which Ric-8A catalyzes these activities, which are stimulated by Casein Kinase II phosphorylation, are unknown. We report the structure of the nanobody-stabilized complex of nucleotide-free $\mathrm{G} \alpha$ bound to phosphorylated Ric-8A at near atomic resolution by cryoelectron microscopy and X-ray crystallography. The mechanism of Ric-8A GEF activity differs considerably from that employed by $G$ protein-coupled receptors at the plasma membrane. Ric-8A engages a specific conformation of $\mathrm{G} \alpha$ at multiple interfaces to form a complex that is stabilized by phosphorylation within a Ric-8A segment that connects two G $\alpha$ binding sites. The C-terminus of $\mathrm{G} \alpha$ is ejected from its beta sheet core, thereby dismantling the GDP binding site. Ric-8A binds to the exposed $G \alpha$ beta sheet and switch II to stabilize the nucleotide-free state of $\mathrm{G} \alpha$.

\footnotetext{
${ }^{1}$ Center for Biomolecular Structure and Dynamics, University of Montana, Missoula, MT 59812, USA. ${ }^{2}$ Department of Bioengineering and James H. Clark Center, Stanford University, Stanford, CA 94305, USA. ${ }^{3}$ Division of Biological Sciences, University of Montana, Missoula, MT 59812, USA. ${ }^{4}$ Macromolecular Crystallography Group, Stanford Synchrotron Radiation Light Source, SLAC National Accelerator Laboratory, Stanford University, Stanford, CA 94025, USA. ${ }^{5}$ Structural Biology Brussels, Vrije Universiteit Brussel (VUB), Brussels, Belgium. ${ }^{6}$ VIB-VUB Center for Structural Biology, VIB, Brussels, Belgium.

7 Department of Pharmacology, University of Michigan Medical School, Ann Arbor, MI 48109, USA. ${ }^{8}$ Biosciences Division of CryoEM and Bioimaging, SSRL, SLAC National Accelerator Laboratory, Stanford University, Menlo Park, CA 94025, USA. ${ }^{9}$ Graduate Program in Biochemistry and Biophysics, University of Montana, Missoula, MT 59812, USA. ${ }^{10}$ Present address: Regeneron Pharmaceutical, Inc., Tarrytown, NY, USA. ${ }^{11}$ These authors contributed equally: Levi J. McClelland, Kaiming Zhang, Tung-Chung Mou. ${ }^{凶}$ email: wahc@stanford.edu; Stephen.sprang@umontana.edu
} 
ic-8A is a cytosolic guanine nucleotide exchange factor (GEF) that activates heterotrimeric G protein alpha subunits $(\mathrm{G} a)^{1,2}$. As a chaperone required for $\mathrm{Ga}$ biogenesis and membrane localization, Ric- 8 homologs are essential to life in multicellular eukaryotes ${ }^{3-5}$. Genetic ${ }^{6-8}$ and biochemical data ${ }^{9}$ support a role for Ric-8A in G protein-coupled receptor (GPCR)independent regulation of asymmetric cell division that is essential for embryonic development ${ }^{10}$. Ric-8A exhibits GEF and chaperone activity towards $\mathrm{Ga}$ of the $\mathrm{i}, \mathrm{q}$, and $12 / 13$ classes $^{1}$, while Ric-8B performs these functions for Gas and Gaolf-each in a variety of cellular contexts ${ }^{11,12}$. Both GEF and chaperone activities are stimulated by Casein Kinase II phosphorylation ${ }^{13}$. That the Ga-class specificity of Ric-8A and Ric- $8 \mathrm{~B}$ is the same for both GEF and chaperone activities ${ }^{14}$ suggests a common mechanistic basis for the two activities.

Ric-8A adopts an armadillo (ARM)/HEAT repeat domain architecture and is structurally unrelated to GPCRs ${ }^{15}$. Biophysical data show that nucleotide-free Gall is structurally dynamic when bound to Ric- $8 \mathrm{~A}^{16,17}$ and shares some properties with GPCRbound $\mathrm{Ga}^{18}$. Namely, Ric-8A induces rotational dynamics in the $\mathrm{G} a$ helical domain ${ }^{19}$ and binds to the C-terminus of $\mathrm{Ga}^{16,20}$. In contrast to GPCRs, there is evidence that Ric-8A forms extensive interactions with the $\mathrm{Ga}$ switch regions that undergo GTPdependent conformational change $e^{1,17,20-22}$, and with the Ga $\beta$ sheet scaffold ${ }^{17}$. To better understand the structural basis of Ric$8 \mathrm{~A}$ GEF and chaperone activity, we have determined the structure of the Ric-8A:Gail complex, using both cryo-electron microscopy (cryo-EM) and X-ray crystallography.

\section{Results}

Cryo-EM and X-ray crystallographic analysis of Ric-8A:Gail. To form the complex we used the N-terminal 491-residue fragment of rat Ric-8A, which is a more active GEF than the fulllength (530 amino acid) protein and, to reduce conformational heterogeneity, we used the N-terminal 31-residue truncation mutant of rat Gail ( $\triangle 31 \mathrm{NGai1})$, an efficacious substrate for Ric- $8 A^{16}$. Recombinant Ric- 8 A(1-491) was phosphorylated by Casein Kinase II at S435 and T440, which is necessary and sufficient for stimulation of GEF activity ${ }^{13}$ (Supplementary Fig. 1). Hereafter we refer to phosphorylated Ric-8A(1-491) as Ric-8A and $\Delta 31$ Nail as Ga.

To stabilize and limit the dynamics of the Ric-8A:Ga complex for crystallographic and cryo-EM experiments, we developed a panel of camelid nanobodies $(\mathrm{Nb})^{23}$ that specifically recognize either Gai1, Ric-8A, or the complex of the two. We formed a series of Ric-8A:Ga: Nb complexes that included from one to four $\mathrm{Nbs}$ from this panel. The quality and resolution of cryo-EM reconstructions derived from these complexes was improved in step with the number of Nbs in the complex. We determined the cryo-EM structure of a complex of Ric-8A:Ga with three Nbs bound to Ric-8A and one bound to the helical domain of Ga. Together, these Nbs do not significantly affect Ric-8A GEF activity (Supplementary Fig. 2). The structure was determined from 327,493 particles with a sufficient orientation distribution derived from 8468 movie images, yielding a $3.9 \AA$ resolution reconstruction (Fig. 1, Supplementary Figs. 3, 4, 5a and Supplementary Table 1).

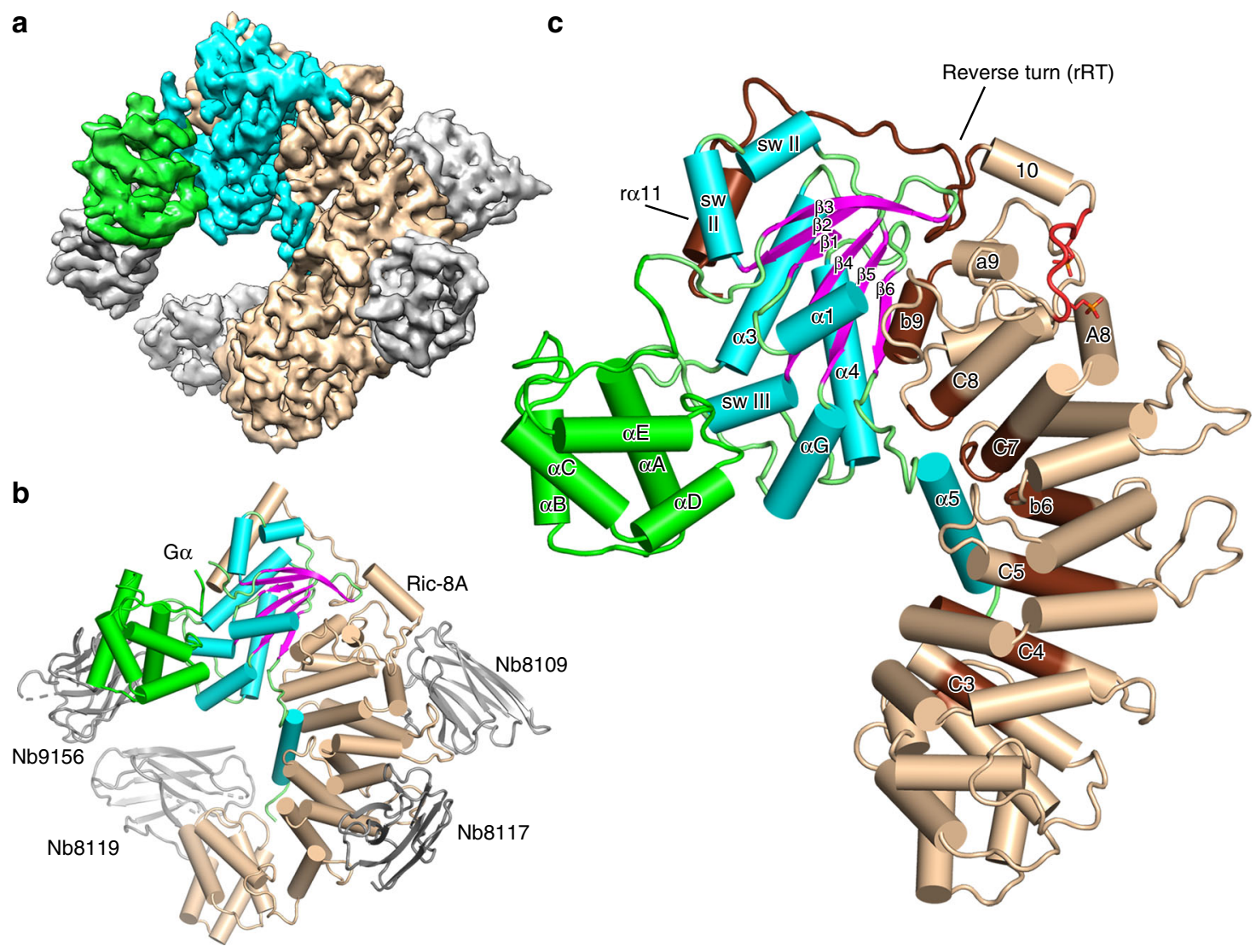

Fig. 1 Architecture of the Ric-8A:Go:4Nb complex. a Cryo-EM 3D map of Ric-8A:G $4: 4 N b$ is shown with Ric-8A colored wheat, G $\alpha$ GTPase and Helical domains colored cyan and green, respectively, and nanobodies colored gray. $\mathbf{b}$ Annotated ribbon and cylinder drawing, colored as in $\mathbf{a}$, but with the $\beta$-strands of $\mathrm{G} \alpha$ rendered in magenta. c The Ric-8A:Goi1:4Nb complex is shown with the nanobodies removed and the loop segments of the G $\alpha$ GTPase domain colored green. Segments of Ric-8A that contact $\mathrm{G} \alpha$ are rendered in dark brown. Ric-8A residues 335-340, which include the two phosphorylation sites are rendered in red. 
We obtained crystals of Ric-8A:Ga bound to the three Ric-8Aspecific Nbs used to generate the cryo-EM structure. Diffraction from these crystals was highly anisotropic (Supplementary Fig. 5b), extending to $4.6 \AA$ along $a *$, and $b *$, and $3.3 \AA$ along $c *$, affording measurement of a $90 \%$ complete anisotropic dataset (Supplementary Table 2). Initial crystallographic phases were determined by molecular replacement and subsequently used to fit the cryo-EM density map. Iterative cycles of model-building and refinement utilized both cryo-EM and X-ray diffraction data to generate the final models (Supplementary Table 1 and 2). In the following discussion, we use prefixes " $r$ " and " $g$ " for residue and secondary structure identifiers of Ric-8A and Ga, respectively. ARM/HEAT repeat helices of Ric- $8 \mathrm{~A}$ are designated according to their position in the repeat: " $A$ ", " $B$ " or " $C$ " for ARM repeats or " $a$ " and " $b$ " for HEAT repeats, followed by the sequence number of the repeat (1 through 9). We use the established nomenclature for $\mathrm{Ga}$ secondary structure $2,24,25$. Descriptions of the crystal structure of Ric-8A:Ga refer to chains $\mathrm{A}$ and $\mathrm{B}$, the better ordered of the two complexes in the asymmetric unit.

The cryo-EM reconstruction reveals Ric-8A residues $2-487$ and the whole of $\mathrm{Ga}$ with the exception of the disordered linker (residues 50-76) between the Helical and GTPase domains (Fig. 1a, b and Supplementary Movie 1). The crystal structure of the Ric-8A:Ga complex also reveals continuous electron density for Ric-8A except for the linkage between 422 and 430. Notably, residues C-terminal to the last HEAT repeat ( $\mathrm{r} 430-\mathrm{r} 491)$ are disordered in structures of Ric-8A in which $\mathrm{Ga}$ is absent ${ }^{15,20}$. In the crystal structure, which lacks Nb9156, the helical domain of $\mathrm{G} \alpha$ and its connections to the GTPase domain, including much of gal and all of switch I, are disordered. Otherwise the cryo-EM and X-ray models of Ric-8A:Ga are in good agreement, although certain loop regions in both $\mathrm{Ga}$ and Ric-8A show significant divergence (Supplementary Fig. 6). Small angle X-ray scattering (SAXS) measurements of the complex are consistent with the $\mathrm{X}$-ray and cryo-EM structures (Supplementary Fig. 7).

Ric-8A displaces the Ga C-terminus and induces GDP release. Binding of Ric-8A to the GTPase domain of $\mathrm{Ga}$ at three noncontiguous contact surfaces bury more than $3200 \AA^{2}$ of solventaccessible surface area (Fig. 2a). Together, these interactions destabilize the guanine nucleotide-binding site (Fig. 3). At the core of the complex, Ric-8A ab9 (r411-r415) and the reverse turn r451-r457 (rRT) interact with the g $\beta 4-\gamma \beta 6$ strands of Ga (Fig. $2 b$ and Supplementary Fig. $8 \mathrm{a}$ and $\mathrm{b}$ ).

Ric-8 ab9 occupies the site of ga5 in GDP-bound Ga, and consequently ga5 is ejected from the concave surface of the $\mathrm{Ga}$ $\beta$-sheet (Fig. 3a). Ric-8A residues rY412, rA415, rA416, and rL418 in rab9 substitute for nonpolar residues of ga5, to stabilize the hydrophobic surface of the $\mathrm{G} \alpha \beta$-sheet core. These residues are conserved in both $\mathrm{A}$ and $\mathrm{B}$ isoforms of vertebrate Ric-8 (Supplementary Fig. 9a), and form Van der Waals interactions with residues in $g \beta 4-g \beta 6$ that are conserved in both Gai and Gas families (Supplementary Fig. 9b). The rW415A mutation severely impairs GEF activity (Fig. 4), although the Y412A mutation does not. At the same interface, steric interactions with raa9 lever the antiparallel g $\beta 2-g \beta 3$ hairpin away from the GTPase core (Fig. 3a). As a unit, $g \beta 1-\mathrm{g} \beta 5$ undergo a $\sim 5^{\circ}$ counter-clockwise rotation as viewed from the concave surface of the $\mathrm{Ga} \beta$-sheet. Changes in the orientation of $g \beta 1-g \beta 3$, in particular, destabilize and induce partial disorder in gal. This, as described below, triggers separation of the Helical and GTPase domains of Ga.

The $\sim 90^{\circ}$ rotation of a5 away from the GTPase domain core (Fig. 3a) reconfigures the "TATC" motif in the $g(\beta 6-\alpha 5)$ GDP purine-binding loop (Fig. $3 \mathrm{~b}$ ). This structural change perturbs the conserved NKKD motif (in $g \beta 4-\alpha G$ ) that confers specificity for guanosine nucleotides ${ }^{26}$. Displacement of these two loops dismantles the GDP purine-binding site. The hydrophobic contact between the N-terminus of ga4 and the Ric-8A aB8aC8 turn (Supplementary Fig. 10) is also important, as indicated by the impairment of GEF activity resulting from the rL383E mutation (Fig. 4).

Binding of the Ga C-terminus to the Ric-8A ARM repeat trough. After its ejection from the $\mathrm{Ga} \beta$-sheet, ga5 is accommodated in a broad trough formed by helices rab2 through raB8 that line the concave surface of ARM/HEAT superhelix of Ric-8A (Fig. 2e and Supplementary Fig. $8 \mathrm{~g}$ and $\mathrm{h}$ ), as observed also in the structure of Ric-8A bound to the C-terminus of transducin ${ }^{20}$. Indeed, after superposition of the respective Ric-8A models, the main-chain and side-chain atoms of the C-termini of transducin (residues 334-350) and Gail(338-354) align with an RMS deviation of $0.36 \AA$. The predominantly hydrophobic amino acid residues of ga5 that interact with the Ric-8A trough (e.g. gF336, gV339, gI343, gI344, and gL348) otherwise pack against the GTPase domain $\beta$-sheet of nucleotide-bound $\mathrm{Ga}^{27}$. Most of the Ric-8A residues that contact ga5 are conserved in Ric-8B (Supplementary Fig. 9a), whereas several of the Ric-8A-contacting residues in ga5 are not conserved among $\mathrm{Ga}$ classes (Supplementary Fig. 9b).

Phosphorylation of Ric-8A stabilizes its interface with Ga. Ric$8 \mathrm{~A}$ phosphorylation acts as an entropic clamp to promote GEF activity. The Ric-8A ab9 and RT segments that interact with the $\mathrm{Ga} \beta$-sheet are connected by an intrinsically disordered sequence, $\mathrm{r} 430-\mathrm{r} 450^{15}$ followed by ra10. The segment spanning $\mathrm{r} 430-\mathrm{r} 440$ is rich in acidic amino acids, and binds within a basic groove formed by raA8 (r344-r358) and raa9 (r401-r410) (Fig. 2c). Phosphorylated rpS435 and rpT440, which are well-ordered (Supplementary Fig. 8i-1) and form multiple ion-pair interactions with conserved lysine and arginine side chains in the Ric-8A electropositive groove, help to immobilize the $\mathrm{r} 430-\mathrm{r} 450$ connector and consequently stabilize rab9 and rRT that interact with $g \beta 4-g \beta 6$. Accordingly, we found earlier that charge $\rightarrow$ neutral mutations of rR345Q and rK349A, which interact with rpS435 and adjacent acidic residues, reduce both basal and phosphorylation-stimulated GEF activity of Ric- $8 \mathrm{~A}^{15}$.

Ric-8A interactions with Ga Switch II and a3. Ric-8A helix a11 (r471-r491), which is preceded by an elongated peptide (r458-r470) that forms an extended arch over the $\alpha 3-\beta 5$ loop of $\mathrm{Ga}$, packs between Ga Switch II and ga3 (Fig. 2d, and Supplementary Fig $8 \mathrm{c}-\mathrm{f}$ ). In the GTP-bound state of $\mathrm{Ga}$, these two elements form the Ga effector protein-binding site ${ }^{28}$. The ejection of ga5 from the $\mathrm{Ga} \beta$-sheet together with interactions between $\alpha 11$ and the Switch II and ga3 interfaces was recently deduced from steered molecular dynamics calculations consistent with small angle X-ray scattering and crosslinking data ${ }^{22}$. ra11 occupies the position of Switch II in the G protein heterotrimer ${ }^{29}$, and also in Gail bound to GTP analogs ${ }^{27}$ and in the Gail $\bullet$ GDP•Pi product complex $^{30}$ (Fig. 3c). However, Switch II is disordered in Gail•GDP and probably also in nucleotide-free $\mathrm{Ga}^{25}$. Ric-8A interactions with switch II are functionally important, since mutations of two $\mathrm{Ga}$-contacting residues in ra11, rE478, and L482, which are conserved in both Ric-8A isoforms, impair GEF activity (Fig. 4). The conformations of Switch II observed in the Xray and cryo-EM structures differ from each other, and from those adopted in nucleotide-bound $\mathrm{Ga}^{28}$ (Supplementary Fig. 8e, f). 

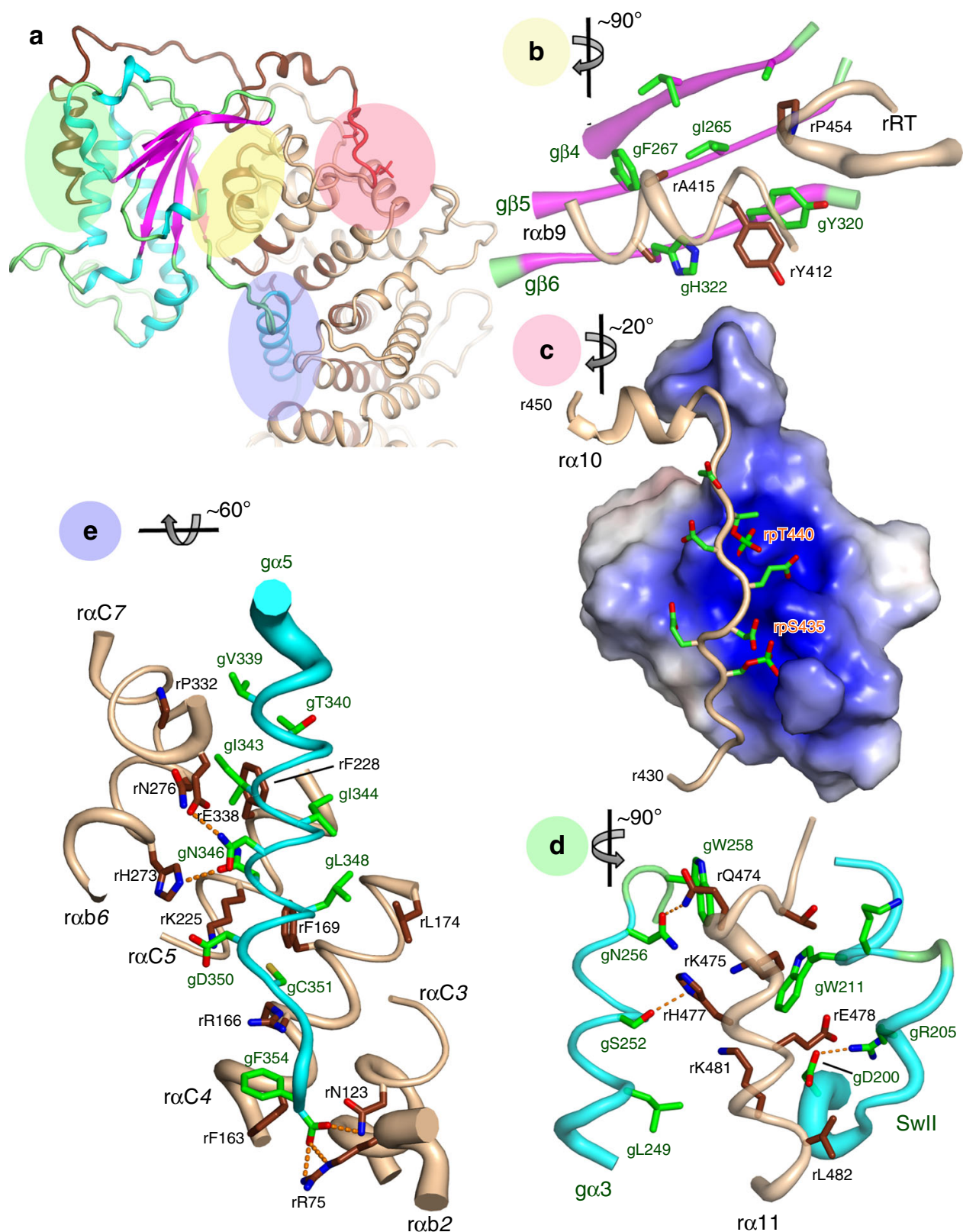

Fig. 2 Interactions of Ric-8A with Goi1. Three major Ric-8A:G $\alpha$ contact surfaces and phosphorylation sites are highlighted in the green, yellow, pink, and blue overlays (panel $\mathbf{a}$ ) and enlarged in panels $\mathbf{b}$-e. Axes adjacent to each panel label indicate the rotation applied to the overall schematic to generate the panel view. $\mathbf{b}$ Interaction between $\mathrm{G} \alpha \beta$-sheet and the Ric-8A C-terminal ARM/HEAT repeat helix r $\alpha b$ A9 and reverse turn $r 451-r 457$ ( $r$ RT) from the crystal structure. Polypeptide backbones are rendered as tubes, with diameter proportional to $B$-factor at $C \alpha$. Carbon atoms of Ric- 8 A and Goi1 are rendered in deep brown, and green, respectively, and oxygen and nitrogen atoms are, respectively, colored red and blue. c Acidic Ric- $8 \mathrm{~A}$ peptide with phosphorylated residues rpS335 and rpT440 bound to the positively charged surface formed by the 8th and 9th Ric-8A ARM/HEAT repeats from the crystal structure. d Interaction of r $\alpha 11$ with g $\alpha 3$ and Switch II from cryo-EM. Putative hydrogen bonds (donor-acceptor contact $<3.5 \AA$ ) are depicted with orange dashed lines. e Contacts between g $\alpha 5$ and residues in successive ARM/HEAT repeats of Ric-8A, from the crystal structure.

Remodeling of Switch II may be facilitated by Ric-8A-induced displacement of $\beta 2-\beta 3$ (Fig. 3a) described above.

Ric-8A-induced reorientation of the Ga helical domain. Displacement of ga 5 and reorientation of $g \beta 2$-g $\beta 3$ eliminates a nexus of stabilizing interactions with the C-terminal residues of gal, which become disordered (Fig. 3a). As a consequence, contacts between the GTPase and helical domains of $\mathrm{Ga}$ are disrupted. As observed in the cryo-EM structure, the helical domain undergoes a counter-clockwise rotation of $\sim 60^{\circ}$ around an axis roughly aligned with $\alpha \mathrm{D}$ of the $\mathrm{Ga}$ helical domain (Fig. 3d). By this motion, a channel opens between the helical and GTPase domains, providing a path of egress for the nucleotide. The magnitude of the rotation is less than that observed in crystal and cryo-EM structures of GPCR:G protein complexes ${ }^{18,31}$, or deduced from double electron-electron resonance studies of Ric$8 \mathrm{~A}: \mathrm{Ga}^{19}$, possibly because it is limited by steric interactions between the Nbs bound to $\mathrm{Ga}$ and Ric-8A. The variability in the orientation of the helical domain is also suggested by normal-mode analysis of the SAXS data (Supplementary Fig. 7). The coupled interactions between Ric-8A and Gail, described above, induce profound conformational changes that dismantle the $\mathrm{G} \alpha$ guanine nucleotidebinding site (Fig. 3b and Supplementary Movie 2). 
a

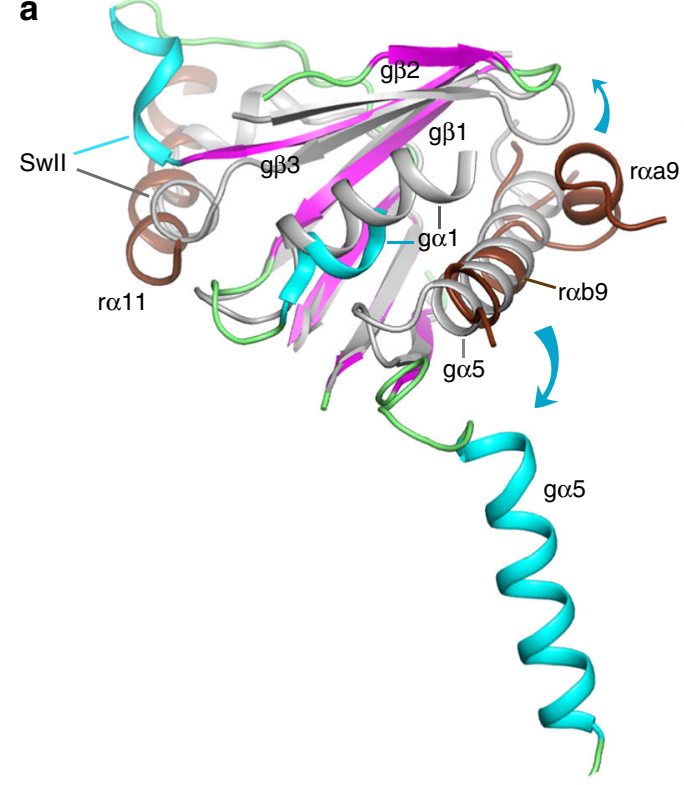

C

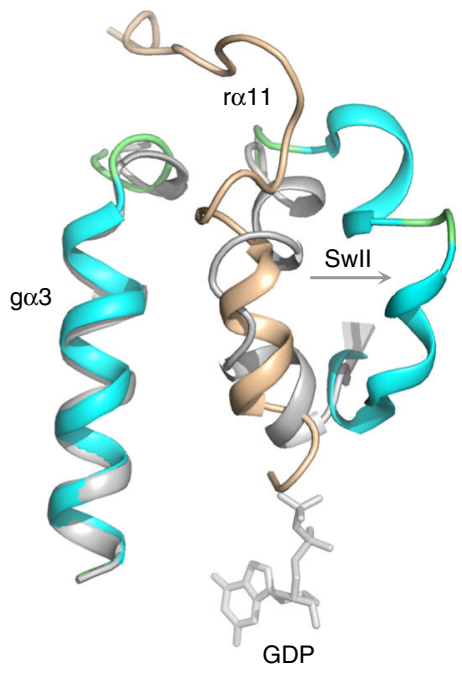

b

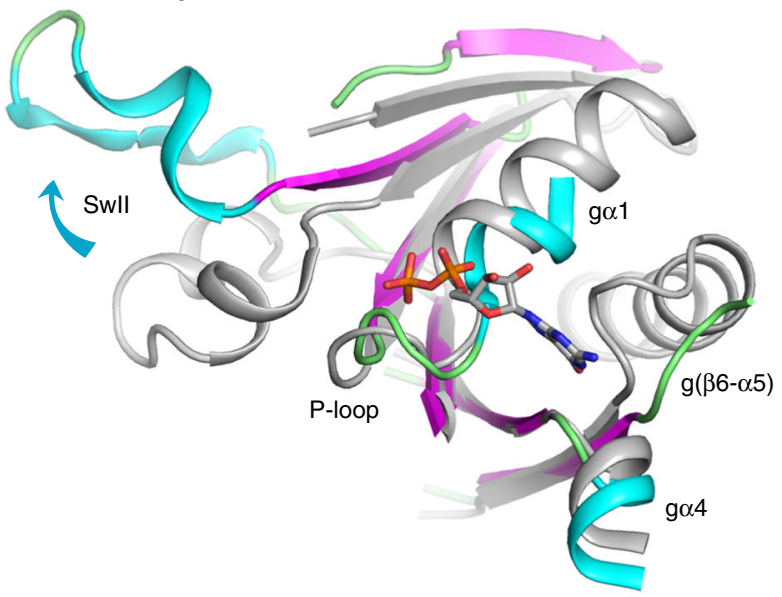

d

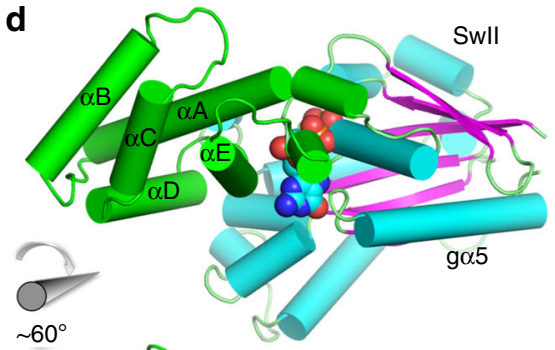

$\sim 60^{\circ}$

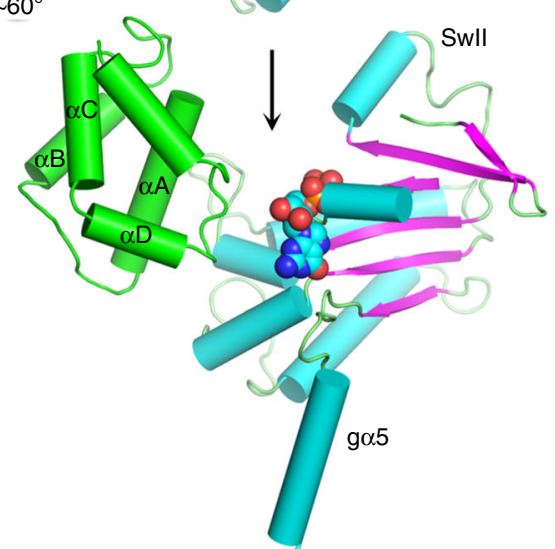

Fig. 3 Conformational changes induced in Go by its interaction with Ric-8A. In panels a-c, GDP•Pi-bound Goi1 (PDB ID 1GIT) [https://doi.org/10.2210/

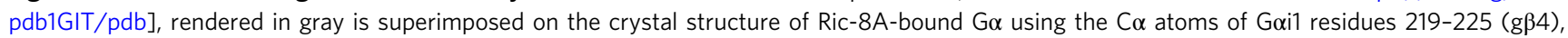

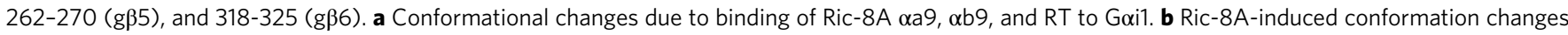
dismantle the G $\alpha$ nucleotide-binding site (see text). GDP from 1GIT is included as a stick model for reference. c Displacement of Switch II by r $\alpha 11$. The

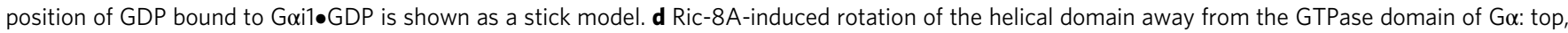
Goil•GDP (1GIT) rendered with helices as cylinders and $\beta$-strands as ribbons and the helical domain colored green; atoms of GDP are rendered as spheres. Bottom, the cryo-EM-derived model of Ric-8A-bound G $\alpha$ with GDP from 1GIT shown as a reference point.

\section{Discussion}

The basis for the Ga class selectivity of Ric- 8 isoforms is not readily apparent. The great majority of residues at the Ga contact residues are conserved in Ric-8A and Ric-8B (Supplementary Fig 9a). Ga residues that interact with raB9 and rRT are conserved in the Gai and Gas classes. However, several residues in ga5 differ between Gas and Gai classes (Supplementary Fig. 9b), and, as suggested ${ }^{20}$, the latter may engage in more productive interactions with Ric-8A than their Gas counterparts.

The structure of the complex suggests mechanisms that produce the chaperone activity of Ric- $8 \mathrm{~A}$, by which it promotes folding and stabilization of nucleotide-free $\mathrm{Ga}^{3}$. It is remarkable that the magnitude of conformational changes induced in Ga by
Ric-8A far exceed those sufficient to effect GDP release in GPCR-G protein complexes ${ }^{2,18}$. Comparison of the Ric-8A:Ga complex with that of the Gai2: $\beta_{1} \gamma_{2}$ heterotrimer bound to the $A_{1}$ adenosine receptor ${ }^{32}$ (Supplementary Fig. 11) shows that both GEFs induce conformational changes or disorder within the Ga P-loop and in gal. Binding within the transmembrane cavity of GPCRs, the C-terminus of Ga (ga5) undergoes a $60^{\circ}$ rotation and $5 \AA$ displacement ${ }^{18}$, thereby inducing rearrangement of g $\beta 6$-ga 5 and, to a lesser extent, $g \beta 4-\alpha G$, both of which are purine recognition elements. The destabilization of gal by loss of contacts with ga5 is transmitted both to the P-loop and the hinge between the Ras and helical domains, permitting the release of contacts between the two ${ }^{2}$. By its wholesale ejection of ga5 from the Ga $\beta$ - 


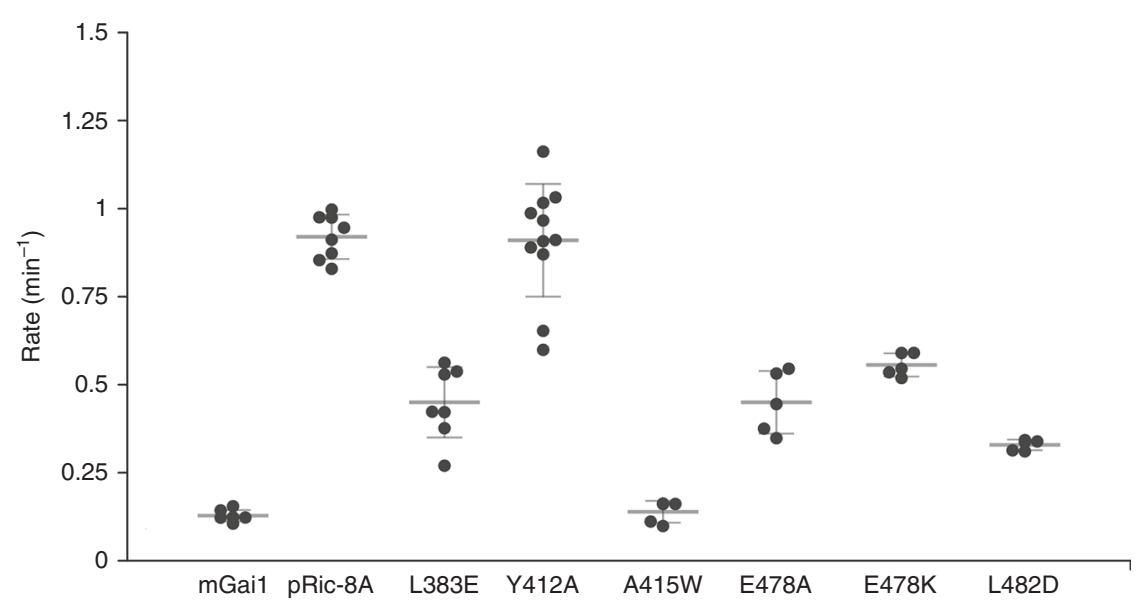

Fig. 4 Mutational analysis of selected residues at the Ric-8A:Go interface. GDP-GTP exchange rates were measured by the rate of tryptophan fluorescence increase upon addition of $\Delta$ N31G $\alpha i 1$ to Ric-8A and GTP $\gamma$ S at final concentrations of $2 \mu \mathrm{M}$ Ric-8A, $1 \mu \mathrm{M} \Delta \mathrm{N} 31 \mathrm{G} \alpha \mathrm{i}$ and $10 \mu \mathrm{M}$ GTP $\gamma \mathrm{S}$. Student's two-tailed $t$-test probabilities that mutant Ric-8A nucleotide exchange rates fall within the distribution of wild-type Ric-8A $(n=8)$ are: L383E, $n=7$, $p=1 \times 10^{-6} ; \mathrm{Y} 412 \mathrm{~A}, n=11, p=0.83$ (not significant); A415W, $n=4, p=7 \times 10^{-12} ; \mathrm{E} 478 \mathrm{~A}, n=5, p=1.7 \times 10^{-5} ; \mathrm{E} 478 \mathrm{~K}, n=5, p=2.9 \times 10^{-8} ; \mathrm{L} 482 \mathrm{D}$ $n=4, p=6 \times 10^{-9}$. mGai1 represents the intrinsic exchange rate for myristoylated Goi1 $\left(n=5, p=5 \times 10^{-10}\right)$. Horizontal bars represent means and 1 standard deviation.

sheet and perturbation of the $\mathrm{Ga} \beta$-sheet itself, which is not observed in interactions with GPCRs, Ric-8A produces the same outcome. Remarkably, the C-terminus of $\mathrm{Ga}$, which tends to disorder, forms anchoring contacts with both GPCRs and with Ric-8A. In contrast, GPCRs do not induce reorientation of the $\mathrm{g} \beta 2-\mathrm{g} \beta 3 \beta$-hairpin, $\beta 1$, or major structural changes in Switch II that are observed in the complex of Ric-8A with Gail.

The large interaction surface formed by Ric-8A with Switch II and the $\mathrm{Ga} \beta$-sheet core may be particularly related to its role as a chaperone. Ric-8A stabilizes nucleotide-free $\mathrm{G} \alpha$ subunits in the absence of $G \beta \gamma^{16}$. Molecular dynamics simulations suggest that contacts between ga5 and the $\mathrm{G \alpha} \beta$-sheet are dynamic in nucleotide-free $\mathrm{Ga}^{33}$. Interactions with Ric-8A would shield these hydrophobic surfaces from exposure in the cytosol. Switch II is also a dynamic structure, even in the GDP-bound state of $\mathrm{Ga}^{26}$ and would likewise be stabilized by Ric-8A a11. Ric-8A(1-491) partially rescues Gail biosynthesis in Ric- $8 A^{-1-}$ cells, but not that of Gaq, for which full-length Ric-8A is required ${ }^{34}$. Hence, the Cterminal $\sim 40$ residues of Ric-8A, which harbor three of the five CKII phosphorylation sites ${ }^{13}$, must play a critical role in Ric-8A chaperone activity. Nevertheless, it is clear that the mechanism by which Ric-8A stabilizes dynamic regions of the nucleotide-free Ga GTPase domain also underlie its GEF activity.

The extensive interface between Ric- $8 \mathrm{~A}$ and $\mathrm{G} \alpha$ prompts the question how the two proteins are able to dissociate upon binding of GTP to Ga, a process that is kinetically facile in comparison to the overall exchange reaction (Fig. 5a and Supplementary Fig. 2). Although displaced slightly by steric conflict with rall (Fig. 3b), the conformation of the P-loop is largely retained in the nucleotide-free $\mathrm{G} \alpha$ bound to Ric-8A (Fig. $5 \mathrm{~b}$ ), thus providing a preformed platform for subsequent binding of GTP. Electrostatic repulsion between the C-terminus of $\mathrm{r} 11$ and the $\gamma$-phosphate of GTP as modeled in Fig. 5b, could promote its release from its binding site between ga3 and Switch II, allowing Switch II to refold into its native GTP-bound conformation. Disruption of Switch II interactions with Ric-8A would restore the native structure of $\mathrm{g} \beta 2-\mathrm{g} \beta 3$, promote its interaction with $\mathrm{g} \alpha 1$ and thereby destabilize the remaining interface of Ric- $8 \mathrm{~A}$ with the GTPase domain. The dynamics that accompany Ric-8A binding to $\mathrm{Ga} \bullet \mathrm{GDP}$ and subsequent release of $\mathrm{Ga} \bullet \mathrm{GTP}$ remain to be explored.

\section{Methods}

Preparation of crosslinked Ric-8A and Goi1. Bis-sulphosuccinimidyl suberate (BS3, Pierce-Thermo-Fisher Scientific) was dissolved in water to a concentration of $100 \mu \mathrm{M}$. K100 reagent (CovalX) is provided as a $2 \mathrm{mg} / \mathrm{ml}$ aqueous solution and contained a proprietary mixture of inert carbon chain spacers (lengths between 8.8 and $13.2 \AA$ ), separating 1-hydroxyl-7-azabenzotriozole groups. Ric-8A:Gail complex, prepared as described ${ }^{16,17,19}$ was dialyzed into $20 \mathrm{mM}$ phosphate buffered saline (PBS) pH 7.4, $1 \mathrm{mM}$ DTT, and final concentration adjusted to $20 \mu \mathrm{M}$ in the same buffer. For each $100 \mu \mathrm{l}$ of complex, $20 \mu \mathrm{l}$ of $\mathrm{K} 100(2 \mathrm{mg} / \mathrm{ml})$ and $25 \mu \mathrm{l}$ of BS3 $(100 \mu \mathrm{M})$ was added, incubated at room temperature for $30 \mathrm{~min}$ and the reaction quenched by addition of $10 \mu \mathrm{l}$ of $1 \mathrm{M}$ Tris $\mathrm{pH}$ 8.5. Samples were centrifuged at $14,000 \mathrm{rpm}$ on a desk-top centrifuge for $10 \mathrm{~min}$ at $4{ }^{\circ} \mathrm{C}$, to remove particulate matter, eluted though a tandem $5 \mathrm{ml}$ HiTrap desalting column (GE Healthcare) to remove excess cross-linking reagents. Proteins were eluted in $20 \mathrm{mM}$ PBS, concentrated to $0.3 \mathrm{mg} / \mathrm{ml}$ and $1 \mathrm{mM}$ fresh DTT added as the final step. Cross-linked samples were analyzed by Coomassie-stained SDS-PAGE and verified by Western blotting using an anti-Ric-8A monoclonal antibody ${ }^{3}$ and mouse anti-Гail monocolonal antibody (Enzo Lifesciences). The crosslinked preparation contained crosslinked Ric-8A:Gail, free Gail, and Ric-8A in roughly equal proportion.

Development of Camelid Nbs. Llamas (Lama glama) were immunized with the cross-linked Ric-8A:Gail preparation. Peripheral blood lymphocytes were isolated from the immunized animals for extraction of total RNA, generation of cDNA and PCR amplification to isolate cDNAs encoding heavy chain variable domains (VHH) for subcloning into the pCTCON2 vector for display on the cell surface of Saccharomyces cerevisiae EBY100 cells, as described ${ }^{23,35,36}$. Cells expressing Nbs that selectively recognize Gail, Ric-8A, or the crosslinked Ric-8A:Gail were identified by three-color FACS sorting on a FACS AriaIII (BD Biosciences), using Dylight 505 (Pierce, Thermo Scientific)-conjugated Ric-8A, Dylight 488-conjugated Gail, and R-phycoerythrin goat anti-mouse antibody (Lucron) to label nanobodyexpressing clones. Three rounds of selection were conducted using $10 \mu \mathrm{M}$ fluorescent proteins in $20 \mathrm{mM}$ PBS pH 7.4, $100 \mathrm{mM} \mathrm{NaCl}$, and $2 \mathrm{mM}$ DTT. Nanobody sequences from selected clones yielding Nbs that exclusively bind to Gail, to Ric8A either free or bound to Gail, or exclusively to the Ric-8A:Gail complex were subcloned in a pMESy4 vector for periplasmic expression in E. coli WK6 cells as described $^{37}$. Nanobody sequences encode a C-terminal hexahistidine affinity tag.

Of the set of $27 \mathrm{Nbs}$ discovered in screening that show strong expression, 20 were capable of binding to either free Ric-8A or to Ric-8A in complex with Gail but not to Gail, three bound exclusively to the Ric-8A:Gail complex and three bound to Gail or its complex with Ric-8A but not to Ric-8A alone. From this set was identified a set of four Nbs capable of binding simultaneously to Ric-8A:Gail, as determined by co-elution as a stable hexameric complex from a GE Healthcare HiLoad 16/600 Superdex $200 \mathrm{pg}$ column and validated by mass spectrometry on a MicroFlex MALDI-ToF MS (Brucker Microflex). Of this set, Nb8109, Nb8117, and Nb8119 bind to Ric-8A and the Ric-8A:Gail complex and Nb9156 binds to Gail.

Nanobody expression and purification. $\mathrm{Nb} 8109,8117,8119$, and 9156 were encoded in pMESy4 vectors for periplasmic expression in WK6 Escherichia coli cells $^{23}$. Briefly, overnight cultures were grown in $50 \mathrm{ml} \mathrm{LB}$ media containing 

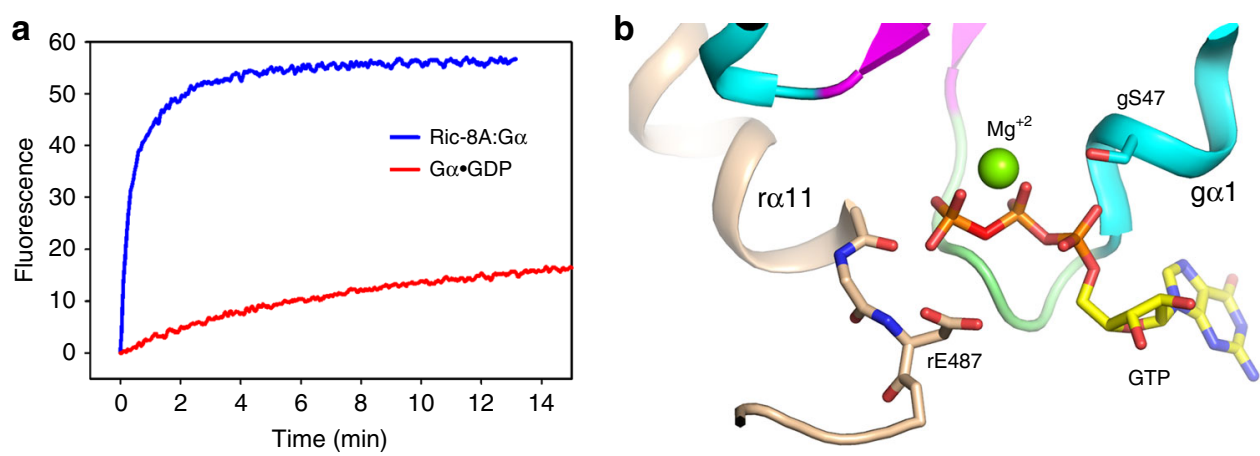

Fig. 5 The P-loop of Goi1 provides a pre-ordered binding site for GTP in the complex with Ric-8A. a Progress curve for GTP (10 $\mu M$ ) binding to purified

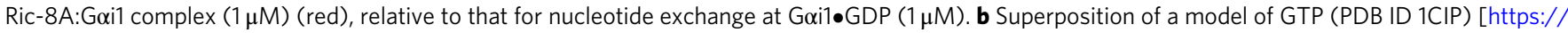

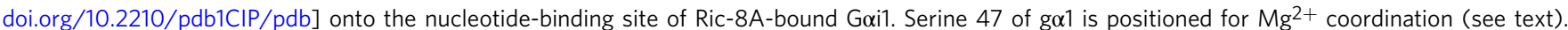

$100 \mu \mathrm{g} / \mathrm{ml}$ Ampicillin, $100 \mathrm{mM}$ Glucose, and $1 \mathrm{mM} \mathrm{MgCl} 2$ in shaker flasks at 190 $\mathrm{rpm}$ and $37^{\circ} \mathrm{C}$. After $\sim 16 \mathrm{~h}$ cells were pelleted at $4000 \mathrm{rpm}(2200 \times \mathrm{g})$ for $10 \mathrm{~min}$ using a benchtop Sorvall Legend RT. Re-suspended pellets were added to $1 \mathrm{~L}$ TB media containing $100 \mu \mathrm{g} / \mathrm{ml}$ ampicillin, $5 \mathrm{mM}$ glucose, and $1 \mathrm{mM} \mathrm{MgCl}$ then incubated at $190 \mathrm{rpm}$ and $37^{\circ} \mathrm{C}$ until achieving an $\mathrm{OD}_{600}$ of $0.7-1.0$, at which point temperature was lowered to $28^{\circ} \mathrm{C}$ and growths were induced with $300 \mu \mathrm{M}$ isopropyl $\beta$-D-1-thiogalactopyranoside (IPTG). Approximately $16 \mathrm{~h}$ post-induction, cells were pelleted at $8000 \mathrm{rpm}(12,000 \times g)$ for $15 \mathrm{~min}$ in a Sorvall RC $6+$ Centrifuge and cell pellets were stored at $-80^{\circ} \mathrm{C}$. Pellets were re-suspended in $100 \mathrm{ml}$ of TES buffer ( $0.2 \mathrm{M}$ Tris $\mathrm{pH} 8,0.5 \mathrm{mM}$ EDTA, $0.5 \mathrm{M}$ sucrose) at $4{ }^{\circ} \mathrm{C}$, vortexed and allowed to stir for a minimum of $1 \mathrm{~h}$. Cells were then added dropwise to a 200 $\mathrm{ml}$ solution of TES/4 buffer ( $0.05 \mathrm{M}$ Tris $\mathrm{pH} 8,0.125 \mathrm{mM}$ EDTA, $0.125 \mathrm{M}$ sucrose), then stirred for $1 \mathrm{~h}$ at $4{ }^{\circ} \mathrm{C}$. Lysate was then spun down at $8000 \mathrm{rpm}(12,000 \times \mathrm{g})$ for $30 \mathrm{~min}$ and supernatant was loaded on a gravity column with Ni-NTA agarose resin (Qiagen). Nanobody-bound resin was washed with 20 column volumes of Wash 1 (0.05 M Tris pH 8, $1 \mathrm{M} \mathrm{NaCl}), 5$ column volumes of Wash 2 (0.05 M MES pH $6,1 \mathrm{M} \mathrm{NaCl}), 10$ column volumes of Wash $3(0.05 \mathrm{M}$ Tris $\mathrm{pH} 8,0.5 \mathrm{M} \mathrm{NaCl})$, and then eluted with 3 column volumes of elution buffer $(0.05 \mathrm{M}$ Tris $\mathrm{pH} 8,0.2 \mathrm{M}$ $\mathrm{NaCl}, 0.5 \mathrm{M}$ imidazole). Eluted nanobody was dialyzed overnight in $0.05 \mathrm{M}$ Tris $\mathrm{pH}$ 8 and $0.2 \mathrm{M} \mathrm{NaCl}$, then concentrated to $5-10 \mathrm{mg} / \mathrm{mL}$ before use or storage at $-80^{\circ} \mathrm{C}$. Before use all $\mathrm{Nb}$ were gel purified using a Superdex 200 10/300 GL sizeexclusion column (GE Healthcare) in gel filtration buffer: $50 \mathrm{mM}$ HEPES $\mathrm{pH} 8$, $150 \mathrm{mM} \mathrm{NaCl}$, and $1 \mathrm{mM}$ TCEP.

Complexes of Ric-8A, $\Delta \mathrm{N} 31 \mathrm{Gai1}$, and $\mathrm{Nb} \mathrm{Nb} 8109, \mathrm{Nb} 8117, \mathrm{Nb} 8119$ in the presence or absence of Nb9156 were formed by incubating Ric-8A, $\Delta$ N31Gail, and $\mathrm{Nb}$ at a 1:2:2 (per nanobody) molar ratio on ice for $16 \mathrm{~h}$ and subsequently purified by gel filtration chromatography using a GE Healthcare HiLoad 16/600 Superdex $200 \mathrm{pg}$ in gel filtration buffer.

G $\alpha$ and Ric-8A protein expression and purification. N-terminally glutathione-Stransferase-tagged Rat Gail with a 31 residue N-terminal truncation $(\Delta \mathrm{N} 31 \mathrm{Gail})$ was expressed from a pDest15 vector in Bl21(DE3) RIPL Escherichia coli and purified as previously described ${ }^{16,38}$. N-terminally hexahistidine-tagged Rat Ric-8A (residues 1-491, hereafter Ric-8A) was expressed and purified as previously described in a pET28 a vector with BL21(DE3) RIPL Escherichia coli cells ${ }^{15-17}$ Following removal of the hexahistidine tag using Tobacco Etch Virus protease, Ric$8 \mathrm{~A}$ protein was loaded onto a Source $15 \mathrm{Q}$ column and eluted with a $500 \mathrm{mM} \mathrm{NaCl}$ gradient at $180 \mathrm{mM} \mathrm{NaCl}$ and subsequently loaded on a GE Healthcare HiLoad 16/ 600 Superdex 200 column for size-exclusion chromatography in gel filtration buffer containing $50 \mathrm{mM}$ Tris $\mathrm{pH} 8,150 \mathrm{mM} \mathrm{NaCl}$, and $1 \mathrm{mM}$ Tris(2-carboxyethyl) phosphine (TCEP).

Ric-8A was phosphorylated using casein kinase II (New England Biolabs) as previously described ${ }^{15,39}$. Briefly, purified Ric-8A was mixed 1:1 with a $2 \mathrm{X}$ kinase reaction buffer containing $0.1 \mathrm{M}$ HEPES pH $8,0.1 \mathrm{M} \mathrm{NaCl}, 20 \mathrm{mM} \mathrm{MgCl}_{2}, 2 \mathrm{mM}$ EGTA, and $1 \mathrm{mM}$ DTT. ATP was added to a final concentration of $5 \mathrm{mM} .160$ Units of casein kinase II were added per mg of Ric-8A, and the reaction was allowed to proceed for $\sim 16 \mathrm{~h}$ at room temperature. Phosphorylated protein was loaded onto a Source $15 \mathrm{Q}$ column pre-equilibrated with $50 \mathrm{mM}$ HEPES pH 8 , $25 \mathrm{mM} \mathrm{NaCl}$, and $2 \mathrm{mM} \beta$-ME, and eluted from a $500 \mathrm{mM} \mathrm{NaCl}$ gradient at $210 \mathrm{mM} \mathrm{NaCl}$. Phosphorylated Ric-8A exhibits an $\sim 2 \mathrm{mS} / \mathrm{cm}$ shift compared to unphosphorylated Ric-8A (Supplementary Fig. 1b). All procedures described hereinafter were conducted with phosphorylated Ric- $8 \mathrm{~A}$ comprising residues $1-491$ of the full-length protein and referred to as Ric-8A. Myristoylated Gail (mGail), used in nucleotide exchange assays, was prepared as described previously ${ }^{15}$. Mutants of Ric-8A, used in nucleotide exchange assays, were constructed via the QuikChange II XL Site-Directed Mutagenesis Kit (Agilent). Expression, purification, and phosphorylation proceeded as described above.
Guanine nucleotide exchange assays. Assays were conducted by measuring the change in Gail tryptophan fluorescence in the presence or absence of Ric-8A using either $\Delta \mathrm{N} 31 \mathrm{Gail}$ or mGail, as described ${ }^{17}$. All proteins were buffer exchanged and assays were conducted in $50 \mathrm{mM}$ HEPES pH $8,150 \mathrm{mM} \mathrm{NaCl}, 10 \mathrm{mM} \mathrm{MgCl}_{2}$, $1 \mathrm{mM}$ TCEP. Experiments were carried out at $20^{\circ} \mathrm{C}$ using a LS55 luminescence spectrometer (Perkin Elmer) with $4 \mathrm{~nm}$ slit widths (excitation $295 \mathrm{~nm}$, emission $345 \mathrm{~nm}$ ). GEF activity for Ric-8A and Ric-8A mutants were measured by preincubating $450 \mu \mathrm{l}$ of Ric-8A and GTP $\gamma \mathrm{S}$ in a quartz fluorescent cuvette prior to addition of $50 \mu \mathrm{l}$ of $10 \mu \mathrm{M}$ mGail for a final concentration of $0.5 \mu \mathrm{M}$ Ric-8A, $1 \mu \mathrm{M}$ mGail, and $10 \mu \mathrm{M}$ guanosine $5^{\prime}$-O-[gamma-thio]triphosphate (GTP $\left.\gamma \mathrm{S}\right)$ in $500 \mu \mathrm{l}$. GTP $\gamma \mathrm{S}$ binding to size-exclusion chromatography purified complexes of Ric-8A: $\triangle \mathrm{N} 31 \mathrm{Gail}$ with and without $\mathrm{Nb}$ were compared to intrinsic nucleotide exchange of $\triangle \mathrm{N} 31 \mathrm{Gail}$. GTP $\gamma \mathrm{S}$ was added to preincubated complexes for final concentrations of $1 \mu \mathrm{M}$ complex (or $\Delta \mathrm{N} 31 \mathrm{Gail}$ ) and $10 \mu \mathrm{M}$ GTP $\gamma \mathrm{S}$. For each assay, a minimum of five technical replicates were taken for each sample; more replicates were performed if the series of assays was conducted over several days, to control for changes in sample activity. Progress curves were fit to single or double (If a slow binding phase was detected) exponential rate models using SigmaPlot 7. Statistical significance of rate differences between reference and test samples was determined by a two-tailed Student's $t$-test. Probability that differences are derived from a random distribution is reported. All data points are shown in box plots that show mean and standard deviation for each data set ${ }^{40}$

Crystallization of the $\triangle$ N31Goi1:Ric-8A:3Nb complex. Crystallization trials for the Ric-8A: $\Delta$ N31Gai1:Nb8109:Nb8117:Nb8119 complex were conducted by vapor diffusion using commercially available crystallization screening kits. Sitting drops were set on 96-2 well INTELLI-PLATEs (Art Robbins Instruments) using a Gryphon crystallization robot (Art Robbins Instruments) at $3-10 \mathrm{mg} / \mathrm{mL}$ protein complex at a $1: 1 \mathrm{v} / \mathrm{v}$ ratio with precipitation solution. Initial crystallization conditions were identified from hits on the ShotGun screen (MD1-88 Molecular Dimensions). Further optimization was carried out by grid screening variations in sodium malonate (Hampton Research) concentration and $\mathrm{pH}$ and by crystal seeding by hanging drop on 24-well VDXm plates (Hampton Research). Crystal seed stocks were prepared with the Seed Bead kit (Hampton Research) in $1.4 \mathrm{M}$ sodium malonate $\mathrm{pH}$ 6.9. $0.9 \mu \mathrm{l}$ of protein stock was added to $0.6 \mu \mathrm{l}$ of reservoir and $0.3 \mu \mathrm{l}$ of crystal seed stock and incubated at $12{ }^{\circ} \mathrm{C}$ for a minimum of $1-2$ weeks. Optimal crystals were obtained from hanging drops containing $3.6 \mathrm{mg} / \mathrm{ml}$ Ric-8A: $\triangle \mathrm{N} 31$ Gail:3Nb, $1.4 \mathrm{M}$ sodium malonate $\mathrm{pH} 6.9$ at $12{ }^{\circ} \mathrm{C}$.

Crystallographic data collection and processing. Diffraction data were measured at the NSLS-II FXS beamline from $\mathrm{LN}_{2}$ cryoprotected crystals measuring $\sim 50 \mu \mathrm{m} \times$ $50 \mu \mathrm{m} \times 5 \mu \mathrm{m}$ employing a helical data collection mode ${ }^{41}$, using X-rays of 0.9793 wavelength. A Si (111) double crystal monochromator was used to focus the X-ray beam to a spot size of $1 \mu \mathrm{m} \times 1 \mu \mathrm{m}$ at the sample. Data were measured in the Phi axis rotation mode in $0.2^{\circ}$ fames, at $0.06 \mathrm{~s} /$ frame at a beam attenuation of 0.7 . Diffraction data were measured on an Eiger $16 \mathrm{M}$ detector at a $133 \mathrm{~Hz}$ frame rate. Due to significant $(>0.5 \AA)$ differences in unit cell axis dimension between crystals, data from a single crystal were selected for data processing and structure determination. In view of the considerable anisotropy of diffraction $(\sim 4.6 \AA$ along $a *$ and $b *$ and $3.3 \AA$ along $c *$ ), three separate data-processing strategies were conducted using the AutoPROC v1.0.2 software toolbox ${ }^{42}$ for evaluation in subsequent model-building and refinement steps. All three utilized $\mathrm{XDS}^{43}$ for data indexing and initial integration. The Standard Isotropic protocol uses SCALEA and TRUNCATE from the CCP4 ${ }^{44}$ to generate isotropic data with a resolution cutoff (4.6 $\AA$ ) determined by the criteria $R_{\text {pim }} \geq 0.6, I / \sigma(I) \geq 2.0$ and $C_{1 / 2} \geq 0.3$. The Extended Isotropic protocol employed POINTLESS and AIMLESS scaling and analysis software ${ }^{45}$ to generate scaled intensities extending to the $3.2 \AA$ resolution 
with $I / \sigma(I) \geq 1.2$. The Anisotropic Filtering protocol implemented in STARANISO $^{46}$ generated a dataset that incorporates intensities within a locally averaged value of $I / \sigma(I)$ to define an anisotropic diffraction cut-off surface (Supplementary Table 2).

\section{Crystallographic model building and refinement. All crystallographic calcula-} tions were conducted using the PHENIX 1.1.6 software package ${ }^{47}$ unless otherwise noted. Initial crystallographic phases for the Ric-8A: $\triangle \mathrm{N} 31$ Gail:3NB complex were determined by Molecular Replacement using atomic coordinates of Ric-8A (residues 1-426; PDB ID:6NMG), the Ras domain of Gail:GDP (PDB ID: 1BOF), and an anti-VGLUT nanobody (PDB ID:5OCL) as search models. An initial atomic model, consisting of coordinates for Ric-8A residues 1-426, residues 34-65 and $190-320$ of $\Delta$ N31Gail and three nanobody backbone models were manually refit into a sigma-weighted $2 \mathrm{mFo}-\mathrm{DFc}$ map using Coot v0.8.6 ${ }^{48}$ and refined using phenix.refine. Completion and refinement of the crystallographic model followed the following general strategy: (1) model-fitting to the cryo-EM reconstruction in regions of the structure in which the path of the polypeptide chain was not defined in (or differed substantially from) the $2 \mathrm{mFo}$-DFc map followed by real space refinement with secondary structure and geometry restraints using phenix.real_space_refine ${ }^{49}$; (2) refitting of the latter to the $2 \mathrm{mFo}-\mathrm{DFc}$ map and subsequent refinement; (3) refinement of the cryo-EM map (see below) using the crystallographic model as an alignment reference. In the regions in which the polypeptide path observed in the cryo-EM-derived and crystallographic models diverged, no attempt was made to bring them into agreement. Initial cycles of crystallographic model building and refinement were carried out using Standard Isotropic or Extended isotropic data sets derived from datasets derived from merging and scaling data from the two most strongly diffracting crystals. The final rounds of fitting and refinement of the complete model (excluding the C-terminus of Ga1, the Helical Domain, and Switch I, residues 51-184) utilized an Anisotropic Supplementary set comprising data from the single crystal that afforded the strongest diffraction and optimal merging statistics (see Supplementary Table 2). A TLS model was applied during refinement using phenix.refine. Model quality and correlation with the refined electron density were performed using MolProbity ${ }^{50}$. The atomic coordinates for the refined crystallographic model for the Ric- $8 \mathrm{~A}$ : $\triangle \mathrm{N} 31 \mathrm{Gail}: 3 \mathrm{Nb}$ complex and associated structure factors are deposited in the RCSB Protein Data Bank ${ }^{51}$ (PDB ID 6TYL). Figures depicting atomic models were rendered using PyMol version 2.3 (Schrodinger, LLC)

Cryo-EM data collection. Three microliters of the Ric-8A: $\triangle$ N31Gail:4Nb complex at $0.4 \mathrm{mg} / \mathrm{ml}$ with $0.01 \%$ NP40 were applied onto glow-discharged 200 -mesh R2/1 Quantifoil grids (Electron Microscopy Sciences). Grids were blotted for $4 \mathrm{~s}$ and rapidly cryocooled in liquid ethane using a Vitrobot Mark IV (Thermo Fisher Scientific) at room temperature and $100 \%$ humidity. The samples were screened using a Talos Arctica cryo-electron microscope (Thermo Fisher Scientific) operated at $200 \mathrm{kV}$ and then imaged in a Titan Krios cryo-electron microscope (Thermo Fisher Scientific) with GIF energy filter (Gatan) at a magnification of $130,000 \times$ (corresponding to a calibrated sampling of $1.06 \AA$ per pixel). Micrographs were recorded using EPU software (Thermo Fisher Scientific) with a Gatan K2 Summit direct electron detector, where each image is composed of 30 individual frames with an exposure time of $6 \mathrm{~s}$ and a dose rate of 11.5 electrons per second per $\AA^{2}$. A total of 8670 movie stacks were collected with a defocus range of -1 to $-3 \mu \mathrm{m}$.

Cryo-EM image processing. All micrographs were motion-corrected using MotionCor $2^{52}$ and the contrast transfer function (CTF) was determined using CTFFIND $4^{53}$. All particles were autopicked using the NeuralNet option in EMAN2 v 2.31 and further checked manually, yielding 768,736 particles from selected 8468 micrographs. Particle coordinates were then imported to Relion v3.0.6, wherein multiple rounds of $2 \mathrm{D}$ classification were performed to remove poor $2 \mathrm{D}$ class averages. Meanwhile, $\sim 20,000$ particle images were selected to build an initial model using the "ab-initio 3D" program in cryoSPARC v2 ${ }^{54}$. A total of 676,130 particles were used for 3D classification in Relion to remove the poor classes. Next, five rounds of $3 \mathrm{D}$ heterogeneous refinement were performed in cryoSPARC to further remove the bad particles. The final 3D refinement with 327,493 particles was performed in cryoSPARC using the "non-Uniform refinement" option with a soft mask of the complex density and a $3.85 \AA$ resolution map was obtained. The resolution of the final map was estimated according to the $0.143 \mathrm{FSC}$ criterion. A $3.9 \AA$ low-pass filter was done to the final 3D map for better display (see more information in Supplementary Fig. 2 and Table 1).

Cryo-EM model building. The cryo-EM model for the Ric-8A: $\Delta$ N31Gail:4Nbs complex was constructed with a refined crystallographic Ric-8A: $\triangle$ N31Gai: $3 \mathrm{Nb}$ model (in which only the GTPase domain was modeled) and a model of the helical domain of Gail in complex with Nb9156 from the crystal structure of Gai:Nb9156 complex (unpublished data). The final Ric-8A: $\triangle$ N31Gail:4Nb cryoEM model was optimized by refinement using phenix.real_space_refine with secondary structure and geometry restraints ${ }^{49}$. Model quality was evaluated with MolProbity 50
Small angle X-ray scattering. SAXS was performed at BioCAT beamline 18ID at the Advanced Photon Source, Chicago, with in-line size exclusion chromatography (SEC-SAXS) to separate sample from aggregates and other contaminants. Protein sample $(\sim 5 \mathrm{mg} / \mathrm{ml}$ in $50 \mathrm{mM}$ HEPES, $\mathrm{pH} 8.0,150 \mathrm{mM} \mathrm{NaCl}$ and $1 \mathrm{mM}$ TCEP $)$ was loaded onto a Superdex 200 Increase 10/300 GL column, which was run at $0.7 \mathrm{ml} /$ min in an AKTA Pure FPLC (GE Healthcare Life Sciences). After detection by an in-line UV monitor, the sample passed through the SAXS flow cell, a $1.5 \mathrm{~mm}$ ID quartz capillary with $10 \mu \mathrm{m}$ walls. Scattering intensity was recorded using a Pilatus 3 $1 \mathrm{M}$ (Dectris) detector which was placed $3.67 \mathrm{~m}$ from the sample affording access to a range of momentum transfer $(q)$ from 0.0065 to $0.35 \AA^{-1}[q=4 \pi \sin (\theta) / \lambda]$. Exposures of $0.5 \mathrm{~s}$ were acquired every $2 \mathrm{~s}$ during elution. Data were reduced using BioXTAS RAW v1.6.0 ${ }^{55}$. Buffer blanks were created by averaging regions flanking the elution peak and subtracted from exposures selected from the elution peak to create the $I(q)$ vs. $q$ curves used for subsequent analyses. Ab initio molecular envelopes were computed by the ATSAS v2.6.2 package ${ }^{56}$ programs DAMMIN ${ }^{57}$. Ten bead models were reconstructed in DAMMIF ${ }^{58}$, which were aligned and averaged in DAMAVER ${ }^{59}$. The Molecular envelope was visualized, and atomic models fit to molecular envelopes using Chimera v1.10.2 ${ }^{60}$. The conformational flexibility of Ric-8A: $\triangle N 31$ Gail was modeled by coarse-grained fitting with respect to experimental SAXS data using SREFLEX program in the ATSAS software package ${ }^{56}$. Normal mode analysis was conducted with automatic determination of rigid body units. The final disposition of rigid body units after application of normal mode projections was determined by rigid body refinement with respect to the computed SAXS profile ${ }^{61}$. CRYSOL software from the ATSAS software package was used to model scattering profiles from atomic coordinates.

Amino acid sequence alignments were conducted using Clustal Omega ${ }^{62}$ via its web-server (https://www.ebi.ac.uk/Tools/msa/clustalo/).

Reporting summary. Further information on research design is available in the Nature Research Reporting Summary linked to this article.

\section{Data availability}

Coordinates of Ric-8A: $\triangle 31$ Gail:4Nb model from cryo-EM are deposited in the RCSB Protein Data Bank (PDB) with ID 6UKT[https://doi.org/10.2210/pdb6UKT/pdb]. Coordinates of Ric-8A: $\triangle 31 \mathrm{Gail}: 3 \mathrm{Nb}$ model from crystal structure are deposited in the PDB with ID 6YTL[https://doi.org/10.2210/pdb6YTL/pdb]. The cryo-EM reconstruction is deposited in the Electron Microscopy Data Bank with id EMD20812[https://www.ebi. ac.uk/pdbe/entry/emdb/EMD-20812]. The small angle X-ray scattering data for the Ric8A: $\triangle 31$ Gail complex is deposited in the Small Angle Scattering Biological Database (SASBDB) ${ }^{63}$ with accession code SASDG95[https://www.sasbdb.org/data/SASDG95]. The source data underlying Fig. 4 and Supplementary Fig. $2 \mathrm{a}$ are provided as a Source Data file. All other relevant data are available from the authors upon reasonable request.

Received: 1 January 2020; Accepted: 10 February 2020; Published online: 26 February 2020

\section{References}

1. Tall, G. G., Krumins, A. M. \& Gilman, A. G. Mammalian Ric-8A (synembryn) is a heterotrimeric Galpha protein guanine nucleotide exchange factor. J. Biol. Chem. 278, 8356-8362 (2003).

2. Flock, T. et al. Universal allosteric mechanism for Galpha activation by GPCRs. Nature 524, 173-179 (2015).

3. Chan, P., Thomas, C. J., Sprang, S. R. \& Tall, G. G. Molecular chaperoning function of Ric-8 is to fold nascent heterotrimeric $\mathrm{G}$ protein alpha subunits. Proc. Natl Acad. Sci. USA 110, 3794-3799 (2013).

4. Hampoelz, B., Hoeller, O., Bowman, S. K., Dunican, D. \& Knoblich, J. A. Drosophila Ric-8 is essential for plasma-membrane localization of heterotrimeric G proteins. Nat. Cell Biol. 7, 1099-1105 (2005).

5. Gabay, M. et al. Ric-8 proteins are molecular chaperones that direct nascent $\mathrm{G}$ protein alpha subunit membrane association. Sci. Signal. 4, ra79 (2011).

6. Miller, K. G. \& Rand, J. B. A role for RIC-8 (Synembryn) and GOA-1 (G(o) alpha) in regulating a subset of centrosome movements during early embryogenesis in Caenorhabditis elegans. Genetics 156, 1649-1660 (2000).

7. Couwenbergs, C., Spilker, A. C. \& Gotta, M. Control of embryonic spindle positioning and Galpha activity by C. elegans RIC-8. Curr. Biol. 14, 1871-1876 (2004).

8. Afshar, K. et al. RIC-8 is required for GPR-1/2-dependent Galpha function during asymmetric division of C. elegans embryos. Cell 119, 219-230 (2004).

9. Woodard, G. E. et al. Ric-8A and Gi alpha recruit LGN, NuMA, and dynein to the cell cortex to help orient the mitotic spindle. Mol. Cell. Biol. 30, 3519-3530 (2010). 
10. Tonissoo, T. et al. Nucleotide exchange factor RIC-8 is indispensable in mammalian early development. Dev. Dyn. 239, 3404-3415 (2010).

11. Chan, P., Gabay, M., Wright, F. A. \& Tall, G. G. Ric-8B is a GTP-dependent G protein alphas guanine nucleotide exchange factor. J. Biol. Chem. 286, 19932-19942 (2011).

12. Hinrichs, M. V., Torrejon, M., Montecino, M. \& Olate, J. Ric-8: different cellular roles for a heterotrimeric G-protein GEF. J. Cell. Biochem. 113, 2797-2805 (2012).

13. Papasergi-Scott, M. M. et al. Dual phosphorylation of Ric-8A enhances its ability to mediate $\mathrm{G}$ protein alpha subunit folding and to stimulate guanine nucleotide exchange. Sci. Signal. 11, https://doi.org/10.1126/scisignal.aap8113 (2018).

14. Papasergi, M. M., Patel, B. R. \& Tall, G. G. The G protein alpha chaperone Ric- 8 as a potential therapeutic target. Mol. Pharm. 87, 52-63 (2015).

15. Zeng, B. et al. Structure, function, and dynamics of the galpha binding domain of Ric-8A. Structure 27, 1137-1147 (2019).

16. Thomas, C. J. et al. The nucleotide exchange factor Ric-8A is a chaperone for the conformationally dynamic nucleotide-free state of Galphail. PLoS ONE 6, e23197 (2011).

17. Kant, R., Zeng, B., Thomas, C. J., Bothner, B. \& Sprang, S. R. Ric-8A, a G protein chaperone with nucleotide exchange activity induces long-range secondary structure changes in Galpha. Elife 5, https://doi.org/10.7554/ eLife.19238 (2016).

18. Rasmussen, S. G. et al. Crystal structure of the beta2 adrenergic receptor-Gs protein complex. Nature 477, 549-555 (2011).

19. Van Eps, N., Thomas, C. J., Hubbell, W. L. \& Sprang, S. R. The guanine nucleotide exchange factor Ric-8A induces domain separation and Ras domain plasticity in Galphai1. Proc. Natl Acad. Sci. USA 112, 1404-1409 (2015).

20. Srivastava, D., Gakhar, L. \& Artemyev, N. O. Structural underpinnings of Ric8A function as a G-protein alpha-subunit chaperone and guaninenucleotide exchange factor. Nat. Commun. 10, 3084 (2019).

21. Nagai, Y., Nishimura, A., Tago, K., Mizuno, N. \& Itoh, H. Ric-8B stabilizes the alpha subunit of stimulatory $\mathrm{G}$ protein by inhibiting its ubiquitination. J. Biol. Chem. 285, 11114-11120 (2010).

22. Srivastava, D. \& Artemyev, N. O. Large-scale conformational rearrangement of the alpha5-helix of Galpha subunits in complex with the guanine nucleotide exchange factor Ric8A. J. Biol. Chem. 294, 17875-17882 (2019)

23. Pardon, E. et al. A general protocol for the generation of Nanobodies for structural biology. Nat. Protoc. 9, 674-693 (2014).

24. Lambright, D. G., Noel, J. P., Hamm, H. E. \& Sigler, P. B. Structural determinants for activation of the alpha-subunit of a heterotrimeric $\mathrm{G}$ protein. Nature 369, 621-628 (1994).

25. Mixon, M. B. et al. Tertiary and quaternary structural changes in Gi alpha 1 induced by GTP hydrolysis. Science 270, 954-960 (1995)

26. Sprang, S. R. G protein mechanisms: Insights from structural analysis. Annu. Rev. Biochem. 66, 639-678 (1997).

27. Coleman, D. E. et al. Structures of active conformations of Gi alpha 1 and the mechanism of GTP hydrolysis. Science 265, 1405-1412 (1994).

28. Sprang, S. R., Chen, Z. \& Du, X. Structural basis of effector regulation and signal termination in heterotrimeric Galpha proteins. Adv. Protein Chem. 74, 1-65 (2007).

29. Wall, M. A. et al. The structure of the $\mathrm{G}$ protein heterotrimer $\mathrm{Gi}$ alpha 1 beta 1 gamma 2. Cell 83, 1047-1058 (1995).

30. Berghuis, A. M., Lee, E., Raw, A. S., Gilman, A. G. \& Sprang, S. R. Structure of the GDP-Pi complex of Gly203->Ala Gia1: a mimic of the ternary product complex of Ga-catalyzed GTP hydrolysis. Structure 4, 1277-1290 (1996).

31. Westfield, G. H. et al. Structural flexibility of the G alpha s alpha-helical domain in the beta2-adrenoceptor Gs complex. Proc. Natl Acad. Sci. USA 108, 16086-16091 (2011).

32. Draper-Joyce, C. J. et al. Structure of the adenosine-bound human adenosine A1 receptor-Gi complex. Nature 558, 559-563 (2018).

33. Dror, R. O. et al. SIGNAL TRANSDUCTION. Structural basis for nucleotide exchange in heterotrimeric G proteins. Science 348, 1361-1365 (2015).

34. Oner, S. S. et al. Regulation of the G-protein regulatory-Galphai signaling complex by nonreceptor guanine nucleotide exchange factors. J. Biol. Chem. 288, 3003-3015 (2013)

35. Chao, G. et al. Isolating and engineering human antibodies using yeast surface display. Nat. Protoc. 1, 755-768 (2006).

36. Young, C. L., Britton, Z. T. \& Robinson, A. S. Recombinant protein expression and purification: a comprehensive review of affinity tags and microbial applications. Biotechnol. J. 7, 620-634 (2012).

37. Abskharon, R. N. et al. Probing the N-terminal beta-sheet conversion in the crystal structure of the human prion protein bound to a nanobody. J. Am. Chem. Soc. 136, 937-944 (2014).
38. Thomas, C. J., Tall, G. G., Adhikari, A. \& Sprang, S. R. Ric-8A catalyzes guanine nucleotide exchange on $\mathrm{G}$ alphail bound to the GPR/GoLoco exchange inhibitor AGS3. J. Biol. Chem. 283, 23150-23160 (2008).

39. Yu, W., Yu, M., Papasergi-Scott, M. M. \& Tall, G. G. Production of phosphorylated Ric-8A proteins using protein kinase CK2. Protein Expr. Purif. 154, 98-103 (2019).

40. Weissgerber, T. L. et al. Data visualization, bar naked: a free tool for creating interactive graphics. J. Biol. Chem. 292, 20592-20598 (2017).

41. Polsinelli, I. et al. Comparison of helical scan and standard rotation methods in single-crystal X-ray data collection strategies. J. Synchrotron Radiat. 24, 42-52 (2017).

42. Vonrhein, C. et al. Data processing and analysis with the autoPROC toolbox. Acta Crystallogr. D 67, 293-302 (2011).

43. Kabsch, W. Xds. Acta Crystallogr. D 66, 125-132 (2010).

44. Collaborative Computational Project, N. The CCP4 suite: programs for protein crystallography. Acta Crystallogr. D50, 760-763 (1994).

45. Evans, P. R. \& Murshudov, G. N. How good are my data and what is the resolution? Acta Crystallogr. D 69, 1204-1214 (2013).

46. Tickle, I. J. et al. STARANISO http://staraniso.globalphasing.org/cgi-bin/ staraniso.cgi (Global Phasing Ltd., Cambridge, UK, 2018).

47. Adams, P. D. et al. PHENIX: a comprehensive Python-based system for macromolecular structure solution. Acta Crystallogr. D 66, 213-221 (2010).

48. Emsley, P., Lohkamp, B., Scott, W. G. \& Cowtan, K. Features and development of Coot. Acta Crystallogr. D 66, 486-501 (2010).

49. Afonine, P. V. et al. Real-space refinement in PHENIX for cryo-EM and crystallography. Acta Crystallogr. D 74, 531-544 (2018).

50. Williams, C. J. et al. MolProbity: more and better reference data for improved all-atom structure validation. Protein Sci. 27, 293-315 (2018).

51. Berman, H. M. et al. The Protein Data Bank. Nucleic Acids Res. 28, 235-242 (2000).

52. Zheng, S. Q. et al. MotionCor2: anisotropic correction of beam-induced motion for improved cryo-electron microscopy. Nat. Methods 14, 331-332 (2017).

53. Rohou, A. \& Grigorieff, N. CTFFIND4: fast and accurate defocus estimation from electron micrographs. J. Struct. Biol. 192, 216-221 (2015).

54. Punjani, A., Rubinstein, J. L., Fleet, D. J. \& Brubaker, M. A. cryoSPARC: algorithms for rapid unsupervised cryo-EM structure determination. Nat. Methods 14, 290-296 (2017).

55. Hopkins, J. B., Gillilan, R. E. \& Skou, S. BioXTAS RAW: improvements to a free open-source program for small-angle X-ray scattering data reduction and analysis. J. Appl. Crystallogr. 50, 1545-1553 (2017).

56. Franke, D. et al. ATSAS 2.8: a comprehensive data analysis suite for smallangle scattering from macromolecular solutions. J. Appl. Crystallogr. 50, 1212-1225 (2017).

57. Svergun, D. I. Restoring low resolution structure of biological macromolecules from solution scattering using simulated annealing. Biophys. J. 76, 2879-2886 (1999).

58. Franke, D. \& Svergun, D. I. DAMMIF, a program for rapid ab-initio shape determination in small-angle scattering. J. Appl. Crystallogr. 42, 342-346 (2009).

59. Volkov, V. V. \& Svergun, D. I. Uniqueness of ab initio shape determination in small-angle scattering. J. Appl. Crystallogr. 36, 860-864 (2003).

60. Pettersen, E. F. et al. UCSF Chimera-a visualization system for exploratory research and analysis. J. Comput. Chem. 25, 1605-1612 (2004).

61. Panjkovich, A. \& Svergun, D. I. Deciphering conformational transitions of proteins by small angle X-ray scattering and normal mode analysis. Phys. Chem. Chem. Phys. 18, 5707-5719 (2016).

62. Sievers, F. et al. Fast, scalable generation of high-quality protein multiple sequence alignments using Clustal Omega. Mol. Syst. Biol. 7, 539 (2011).

63. Valentini, E., Kikhney, A. G., Previtali, G., Jeffries, C. M. \& Svergun, D. I. SASBDB, a repository for biological small-angle scattering data. Nucleic Acids Res. 43, D357-D363 (2015).

\section{Acknowledgements}

This research was supported by NIH R01GM105993 (S.R.S.); NIH P41GM103832, R01GM079429, and S10OD021600 (W.C.); NSF 1738547 (T.-C.M); NIH R01GM088242 (G.G.T.); resources of Instruct-ERIC, part of the European Strategy Forum on Research infrastructures (ESFRI), the Research Foundation-Flanders (FWO) for their support to Nanobody discovery, and the Strategic Research Program (SRP) of the Vrije Universiteit Brussel (S.T. and J.S.). The Integrated Structural Biology Core at the University of Montana Center for Biomolecular Structure and Dynamics is supported by NIH COBRE award P20GM103546 (S.R.S.). We thank Drs. Wuxian Shi and Martin Fuchs at the National Synchrotron Light Source II (NSLS II) for assistance with data collection at the FMX (17-ID-2) beamline, supported by NIH grant P41GM111244 and the Department of Energy (DOE), KP1605010. T.I.D. is supported by the SSRL Structural Molecular Biology Program by the DOE and by NIH grant P41GM103393. Small angle $\mathrm{X}$-ray scattering experiments were conducted at the Advanced Photon Source, operated for the DOE Office of Science by Argonne National Laboratory under Contract No. 
DE-AC02-06CH11357 with support of NIH grant P41 GM103622 and 1S10OD01809001 for purchase of the Pilatus $31 \mathrm{M}$ detector. We thank Dr. Baisen Zeng for assistance with GEF assays and SPR quantitation of Nb-Ric-8A binding.

\section{Author contributions}

L.J.M. prepared and crystallized the Ric-8A:Gail:3Nb complex, assisted with X-ray data collection, conducted mutagenesis studies, and analyzed kinetic data. K.Z. carried out cryo-EM sample screening, image acquisition and processing, generated and refined cryo-EM reconstructions. T.-C.M. fit and refined atomic models to cryo-EM and crystallographic data and conducted SAXS analysis. J.J. prepared and screened samples for cryo-EM data collection, and assisted with data analysis. C.Y-H. Conducted crystallization experiments and assisted with protein preparations. S.L. assisted with cryo-EM data collection and processing. C.J.T. prepared samples for nanobody development and conducted initial nanobody characterization. T.I.D. advised on X-ray data collection and processing. S.T. and A.W. generated nanobodies. G.G.T. provided general consultation on project. J.S. oversaw nanobody development. W.C. oversaw cryo-EM studies. S.R.S. designed project, provided overall supervision, and wrote the manuscript with assistance from co-authors.

\section{Competing interests}

The authors declare no competing interests.

\section{Additional information}

Supplementary information is available for this paper at https://doi.org/10.1038/s41467020-14943-4.

Correspondence and requests for materials should be addressed to W.C. or S.R.S.

Peer review information Nature Communications thanks John H. Kehrl, and the other, anonymous, reviewer(s) for their contribution to the peer review of this work. Peer reviewer reports are available.

Reprints and permission information is available at http://www.nature.com/reprints

Publisher's note Springer Nature remains neutral with regard to jurisdictional claims in published maps and institutional affiliations.

(c) () Attribution 4.0 International License, which permits use, sharing, adaptation, distribution and reproduction in any medium or format, as long as you give appropriate credit to the original author(s) and the source, provide a link to the Creative Commons licence, and indicate if changes were made. The images or other third party material in this article are included in the article's Creative Commons licence, unless indicated otherwise in a credit line to the material. If material is not included in the article's Creative Commons licence and your intended use is not permitted by statutory regulation or exceeds the permitted use, you will need to obtain permission directly from the copyright holder. To view a copy of this licence, visit http://creativecommons.org/ licenses/by/4.0/.

(C) The Author(s) 2020 